\title{
Molecular Dynamic Studies of the Complex Polyethylenimine and Glucose Oxidase
}

\author{
Beata Szefler ${ }^{1, *}$, Mircea V. Diudea ${ }^{2}$, Mihai V. Putz ${ }^{3,4, *}$ and Ireneusz P. Grudzinski ${ }^{5}$ \\ 1 Department of Physical Chemistry, Faculty of Pharmacy, Collegium Medicum, \\ Nicolaus Copernicus University, Kurpinskiego 5, 85-096 Bydgoszcz, Poland \\ 2 Department of Chemistry, Faculty of Chemistry and Chemical Engineering, Babes-Bolyai University, \\ 400028 Cluj-Napoca, Romania; diudea@gmail.com \\ 3 Laboratory of Structural and Computational Physical-Chemistry for Nanosciences and QSAR, \\ Biology-Chemistry Department, West University of Timisoara, Str. Pestalozzi No. 16, \\ 300115 Timisoara, Romania \\ 4 Laboratory of Renewable Energies-Photovoltaics, R\&D National Institute for Electrochemistry and \\ Condensed Matter (INCEMC), Dr. A. Paunescu Podeanu Str. No. 144, 300569 Timisoara, Romania \\ 5 Department of Pharmaceutical Nanotechnology and Nanotoxicology, Faculty of Pharmacy, \\ Medical University of Warsaw, 02-097 Warsaw, Poland; ireneusz.grudzinski@wum.edu.pl \\ * Correspondence: beatas@cm.umk.pl (B.S.); mv_putz@yahoo.com (M.V.P.); \\ Tel.: +48-52-585-36-80 (B.S.); +40-256-592-638 (M.V.P.); Fax: +40-256-592-620 (M.V.P.)
}

Academic Editor: Guido R. M. M. Haenen

Received: 29 July 2016; Accepted: 20 October 2016; Published: 27 October 2016

\begin{abstract}
Glucose oxidase (GOx) is an enzyme produced by Aspergillus, Penicillium and other fungi species. It catalyzes the oxidation of $\beta$-D-glucose (by the molecular oxygen or other molecules, like quinones, in a higher oxidation state) to form D-glucono-1,5-lactone, which hydrolyses spontaneously to produce gluconic acid. A coproduct of this enzymatic reaction is hydrogen peroxide $\left(\mathrm{H}_{2} \mathrm{O}_{2}\right)$. GOx has found several commercial applications in chemical and pharmaceutical industries including novel biosensors that use the immobilized enzyme on different nanomaterials and/or polymers such as polyethylenimine (PEI). The problem of GOx immobilization on PEI is retaining the enzyme native activity despite its immobilization onto the polymer surface. Therefore, the molecular dynamic (MD) study of the PEI ligand (C14N8_07_B22) and the GOx enzyme (3QVR) was performed to examine the final complex PEI-GOx stabilization and the affinity of the PEI ligand to the docking sites of the GOx enzyme. The docking procedure showed two places/regions of major interaction of the protein with the polymer PEI: (LIG1) of $-5.8 \mathrm{kcal} / \mathrm{mol}$ and (LIG2) of $-4.5 \mathrm{kcal} / \mathrm{mol}$ located inside the enzyme and on its surface, respectively. The values of enthalpy for the PEI-enzyme complex, located inside of the protein (LIG1) and on its surface (LIG2) were computed. Docking also discovered domains of the GOx protein that exhibit no interactions with the ligand or have even repulsive characteristics. The structural data clearly indicate some differences in the ligand PEI behavior bound at the two places/regions of glucose oxidase.
\end{abstract}

Keywords: polyethylenimine (PEI); 3QVR; glucose oxidase (GOx); docking; molecular dynamics

\section{Introduction}

Glucose oxidase ( $\beta$-D-glucose: oxygen 1-oxidoreductase, GOx) is an enzyme produced by Aspergillus, Penicillium and other fungi species. It is a dimeric protein composed of two identical subunits (i.e., monomers), each showing two domains: one domain binds to the substrate ( $\beta$-D-glucose) while the other domain binds non-covalently to a cofactor, namely the flavin adenine dinucleotide (FAD), a molecule acting in biological redox reactions. In the GOx enzyme, FAD works as an electron acceptor, being reduced to $\mathrm{FADH}_{2}$, which is finally oxidized by an electron acceptor, e.g., $\mathrm{O}_{2}$, which 
is reduced to hydrogen peroxide $\left(\mathrm{H}_{2} \mathrm{O}_{2}\right)$. The active site of GOx contains three important amino acids involved in this catalytic reaction: His516, and Glu412, which is hydrogen-bonded to His559. Glucose oxidase is a glycoside of mannose carbohydrate (around 16\%) or can be freely isolated, as a non-glycosylated enzyme, from the fungus Phanerochaete chrysosporium. The synthesis of GOx in fungi is promoted by $\mathrm{O}_{2}$, which induces the transcription of the enzyme [1-3]. GOx has become commercially important in the last few years, gaining important use in chemical, pharmaceutical, food, beverage, and other industries. In addition, the gluconic acid produced in the hydrolysis of D-glucono-1,5-lactone has its own important industrial uses [4]. GOx is used as a biosensor (e.g., to measure the concentration of glucose in blood, given the dramatic increase of the number of diabetics in the world). In this respect, the enzyme is immobilized (by a non-covalent bonding) on various nanomaterials and/or chemicals, e.g., carbon nanotubes (CNTs), chitosans, poly( $N$-vinyl imidazole) hydrogels, etc. [5-7]. Recently, a Polish group reported the immobilization of gamma-globulins from human and bovine blood and human polyclonal antibody (IgG) on carbon-encapsulated iron nanoparticles, with magnetic properties [8]. Other authors demonstrated that the activity of two enzymes (R-chymotrypsin and soybean peroxidase) dramatically decreases after their adsorption onto the surface of single-walled carbon nanotubes [9]. The aim of these works is to retain the enzyme native activity despite its immobilization on a rigid surface, e.g., of carbon nanoparticles. A recent molecular dynamics study [10] reported the interaction energy of the SWNT:PSE:GOx complex in the water environment; in parallel, the molecular dynamics performed on the above complex demonstrated the absence of strong interactions between GOx and the nanotube surface. This fact allowed supposing that GOx activity is not changed under immobilization as the SWNT:PSE:GOx complex.

Polyethylenimine (PEI) was intensely studied as a drug delivery system (DDS) in modern anti-cancer therapies [11,12]. PEI gained a large variety of uses, as published to date: in cosmetics as a thickener ingredient (viscosity adjuster), as a very effective polymer for neutralization of the excessive anionic charges (appearing in colloidal suspensions), as a metal chelating agent in corrosion inhibition, a copolymer in solid polymeric electrolytes for electrical energy storage devices, etc. [13,14]. However, there is a little information about the properties of GOx enzyme attached to PEI [15]. This is why, in this work, a molecular dynamics analysis was performed on a PEI structure (PEI_C14N8_07_B22, Figure 1, left) bound to the glucose oxidase enzyme 3QVR (Figure 1, right).
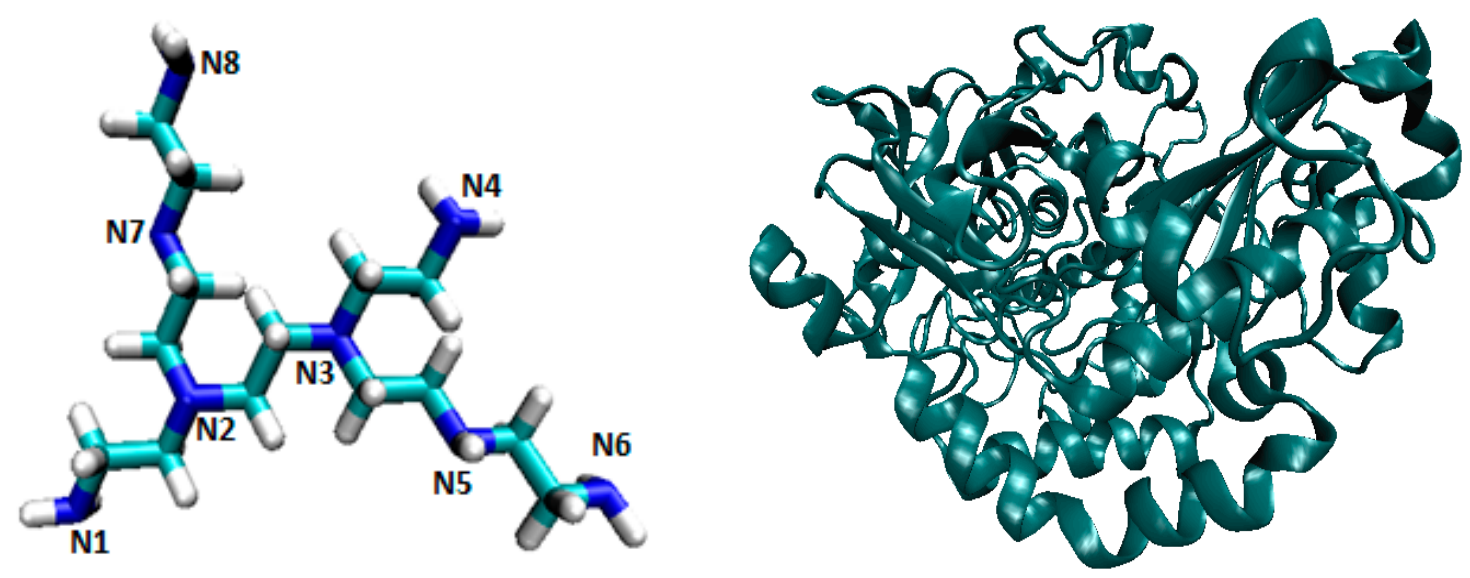

Figure 1. The ligand PEI_C14N8_07_B22 (left) and protein 3QVR (right, Glucose oxidase GOx).

\section{Results and Discussion}

\subsection{Docking Results}

The values of binding free energy data were collected in the vicinity of the protein active site, where the ligand PEI_C14N8_07_B22 was docked; data are given in Tables 1 and 2. 
Table 1. Lamarckian genetic algorithm docked state-Binding energy of ligand PEI_C14N8_07_B22 to the active sites of type A of 3QVR (Glucose oxidase) during the nine explored conformations.

\begin{tabular}{ccccccccccc}
\hline $\begin{array}{c}\text { Ligand } \\
\text { Binding Site }\end{array}$ & $\mathbf{1}$ & $\mathbf{2}$ & $\mathbf{3}$ & $\mathbf{4}$ & $\mathbf{5}$ & $\mathbf{6}$ & $\mathbf{7}$ & $\mathbf{8}$ & $\mathbf{9}$ & $\begin{array}{c}\text { Best Docking } \\
\text { Energy (kcal/mol) }\end{array}$ \\
\hline LIG1 & -5.8 & -5.8 & -5.6 & -5.6 & -5.6 & -5.5 & -5.5 & -5.5 & -5.5 & -5.8 \\
LIG2 & -4.5 & -4.3 & -4.2 & -4.2 & -4.2 & -4.2 & -4.2 & -4.1 & -4.1 & -4.5 \\
LIG3 & -3.8 & -3.8 & -3.7 & -3.6 & -3.6 & -3.6 & -3.6 & -3.6 & -3.5 & -3.8 \\
LIG4 & -3.9 & -3.4 & -2.7 & -1.9 & -1.5 & -1.2 & $\times$ & $\times$ & $\times$ & -3.9 \\
\hline
\end{tabular}

Table 2. Lamarckian genetic algorithm docked state-Binding energy of ligand PEI_C14N8_07_B22 to the active sites of type B of 3QVR during the nine explored conformations.

\begin{tabular}{ccccccccccc}
\hline $\begin{array}{c}\text { Ligand } \\
\text { Binding Site }\end{array}$ & $\mathbf{1}$ & $\mathbf{2}$ & $\mathbf{3}$ & $\mathbf{4}$ & $\mathbf{5}$ & $\mathbf{6}$ & $\mathbf{7}$ & $\mathbf{8}$ & $\mathbf{9}$ & $\begin{array}{c}\text { Docked Energy } \\
\text { (kcal/mol) }\end{array}$ \\
\hline 1 & 0.0 & 0.0 & 0.0 & 0.0 & 0.0 & 0.0 & 0.0 & 0.0 & 0.0 & 0.0 \\
2 & -0.6 & -0.4 & -0.2 & 0.0 & 0.0 & 0.1 & 0.2 & 0.3 & 0.4 & -0.6 \\
3 & 0.8 & 1.6 & 2.4 & 2.7 & 3.0 & $\times$ & $\times$ & $\times$ & $\times$ & 0.8 \\
\hline
\end{tabular}

Based on the values of docked energy: -5.8 and $-4.5 \mathrm{kcal} / \mathrm{mol}$, two sites are differentiated on the protein body, which showed the best affinity to PEI (sites of type A, Table 1). In the first case, the ligand is bound to amino acids inside of the protein area (LIG1, Figure 2), while in the second case the binding takes place outside of the enzyme surface (LIG2, Figure 2). This is why the Molecular Dynamics will be performed for the first and second active places of this protein, separately. In addition, docking demonstrated that there are sites in the protein body that exhibit no interactions ligand-enzyme or even manifest repulsive characteristics (Table 2). The docking study gave information about the type and number of interactions between ligand and amino acid residuals of the enzyme. The results obtained during the docking procedure became the starting point in molecular dynamics study.

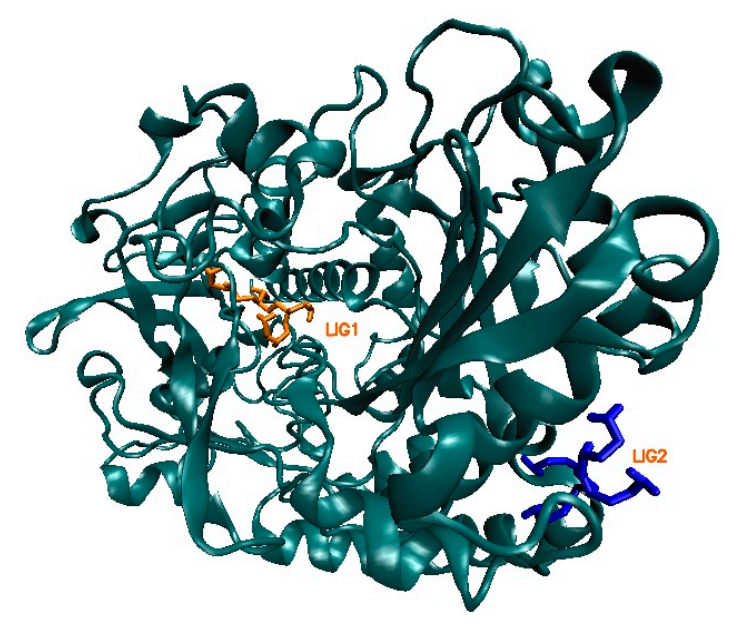

Figure 2. Two sites of the most important interactions ligand-enzyme (3QVR, Glucose oxidase): inside of the protein, named LIG1 and on its surface, named LIG2. As a ligand, the molecule PEI_C14N8_07_B22 was used (see Figure 1, left).

\subsection{Molecular Dynamic Results}

The Molecular Dynamics (MD) simulations allow the study of enzyme-ligand interactions in the natural environment and in a large number of conformations of this complex, as well [16]. During MD, the time evolution of ligand PEI_C14N8_07_B22 lying in two active sites of the conformational space of 3QVR protein, inside (LIG1) and on its surface (LIG2) (Figure 2) was followed. The $60 \mathrm{~ns}$ of collected trajectories were used for structural analysis. In both cases, the first $10 \mathrm{~ns}$ of MD simulation show 
considerable fluctuations (as suggested by RMSD (Root-Mean-Square Deviation) values, Figures 3 and 4), while in the remaining time of simulation, the trajectories seem stabilized, meaning the equilibrium stage was attained. In the case where the ligand interacts inside the protein body (LIG1, Figure 2), the system reached the equilibrium after $10 \mathrm{~ns}$ (Figure 3), while in the second case (ligand outside the enzyme surface (LIG2, Figure 2), after 15 ns (Figure 4).

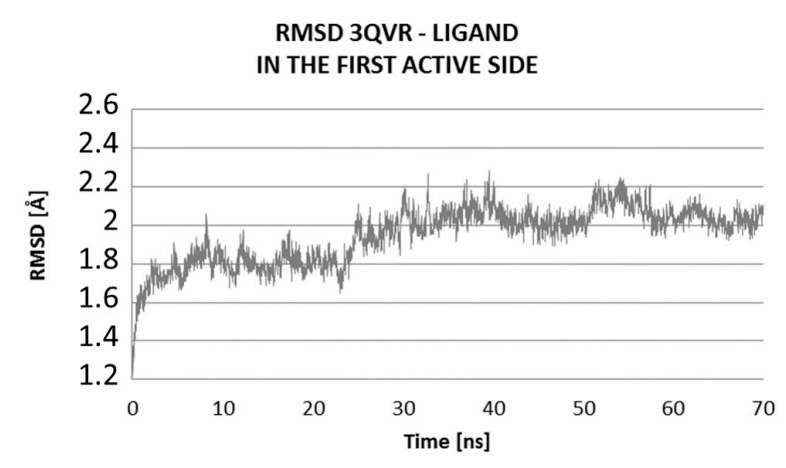

Figure 3. Distribution of RMSD (Root-Mean-Square Deviation) complex protein-ligand: Values characterizing the ligand interaction at the first active site (LIG1) of 3QVR (Glucose oxidase) enzyme, inside of the protein (Figure 2).

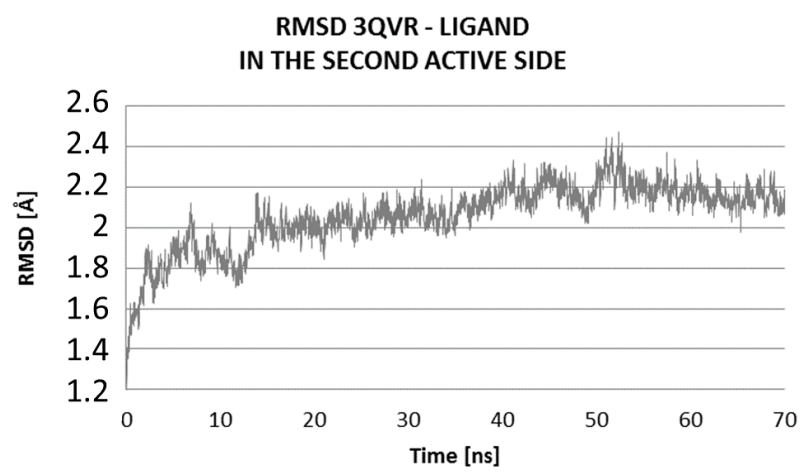

Figure 4. Distribution of RMSD complex protein-ligand: Values characterizing the ligand at the second active site (LIG2) of 3QVR (Glucose oxidase) enzyme (Figure 2).

Comparing the RMSD values of the two systems, their average values and standard deviations, we can see that certainly they are similar, and the difference in average RMSD values is only $0.1 \AA$ (Figure 5, Table 3).

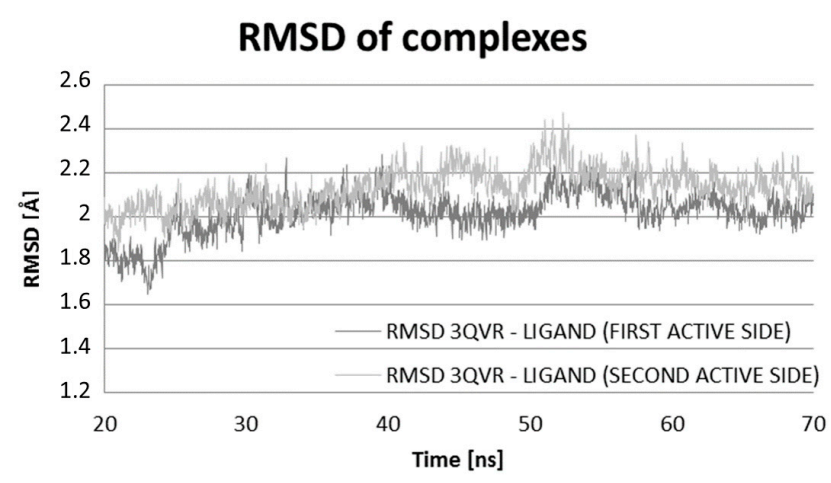

Figure 5. Distribution of RMSD complex protein-ligand: values characterizing the first active site (LIG1) of 3QVR enzyme and the second active site (LIG2) of 3QVR enzyme. The average values of RMSD (A) of the two types of complexes for all trajectories (70 ns) with the standard deviations. 
Table 3. Average RMSDs for the ligand and for the amino acids comprising the active site, across the full MD simulation.

\begin{tabular}{ccccc}
\hline & $\begin{array}{c}\text { Ligand Close to } \\
\text { LIG1 of 3QVR }\end{array}$ & $\begin{array}{c}\text { Ligand Close to } \\
\text { LIG2 of 3QVR }\end{array}$ & $\begin{array}{c}\text { Complex Ligand-Enzyme } \\
\text { at LIG1 Site }\end{array}$ & $\begin{array}{c}\text { Complex Ligand-Enzyme } \\
\text { at LIG2 Site }\end{array}$ \\
\hline RMSD $(\AA)$ & 1.84 & 2.78 & 1.95 & 2.05 \\
SD & 0.24 & 0.30 & 0.14 & 0.16 \\
\hline \multicolumn{4}{c}{ SD $=$ standard deviation. }
\end{tabular}

At the first active side, inside of the protein, after 20 ns of MD (Molecular Dynamic), there is evidence of stabilization of RMSD (Root-Mean-Square Deviation) values of ligand, with poor fluctuations, from 1.6 to $2.0 \AA$ (Figure 6). The average value of RMSD of ligand within the complex at LIG1 side is $1.84 \pm 0.24$, while for the complex with the ligand bound at LIG2 site, is higher, up to $2.78 \pm 0.30 \AA$, suggesting a significantly higher mobility of ligand on the protein surface in comparison to the inside of enzyme (Table 3).

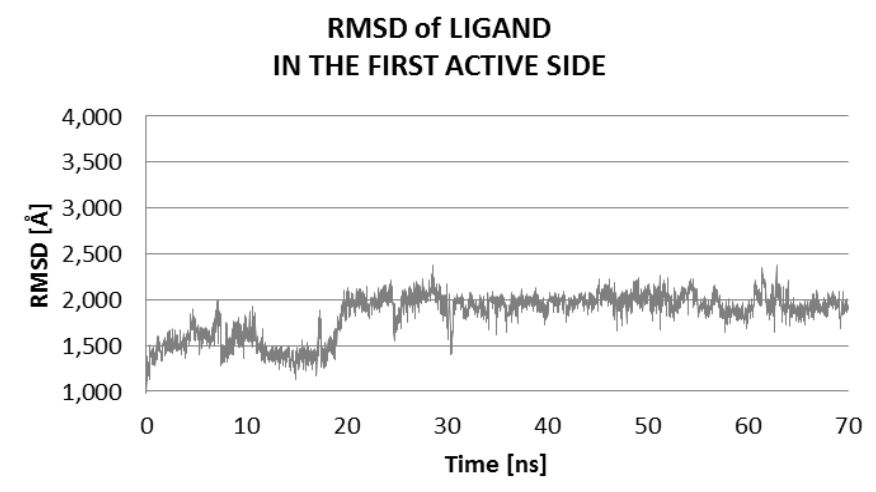

Figure 6. Distribution of RMSD of ligand: values characterizing the ligand interaction at the first active site (LIG1) of 3QVR enzyme, inside the protein (Figure 2).

Behavior of ligand on the surface of protein is similar during all trajectories. Here, the hydrogen bonds are weak, thus they are quickly broken and re-made, cyclically; such weak hydrogen bonds have a length of about $3 \AA$ (Figure 7).

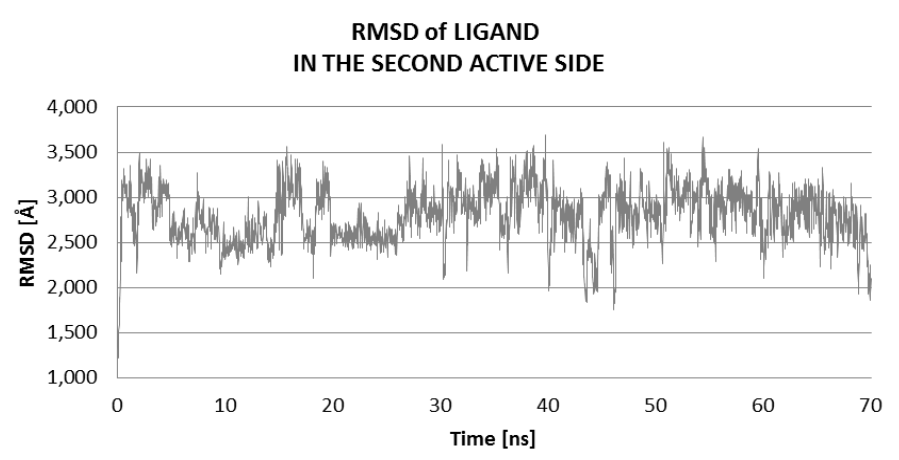

Figure 7. Distribution of RMSD of ligand: values characterizing the ligand interaction at the second active site (LIG2) of 3QVR enzyme, on the surface of protein (Figure 2).

The high fluctuation of the values of RMSD of ligand on the surface of protein during MD was located in the domain of $2 \AA$ to $3.5 \AA$ (Figure 7).

The mobility of the ligand in the active site is correlated with changes in the dihedral angle values (C7-N3-C4-C3, Figure 8), with subsequent fluctuations in stabilization of hydrogen bonds and influence in the strength of hydrogen bonds (amino acid of enzyme $(\mathrm{H}) \ldots . . \mathrm{N}\left(\mathrm{H}_{2}\right)$ ligand). After $20 \mathrm{~ns}$ 
of simulation, there appears a stabilization of the values of dihedral angle of ligand (C7-N3-C4-C3; Figures 6 and 8, with an average value of $-56 \AA$. The stabilization of RMSD values of ligand (Figure 8 , Root-Mean-Square Deviation) is an indication of the creation of hydrogen bonds (HB) between the ligand and enzyme. Inside of the protein, six types of interactions (i.e., bonds) ligand-enzyme (Figures 9 and 10) were found; they are of strong and medium strength (Figure 10). The complex formed on the protein surface also shows six types of interactions (Figures 11 and 12), of a rather poor strength (Figure 12), favorable to hydrogen bond rupture.

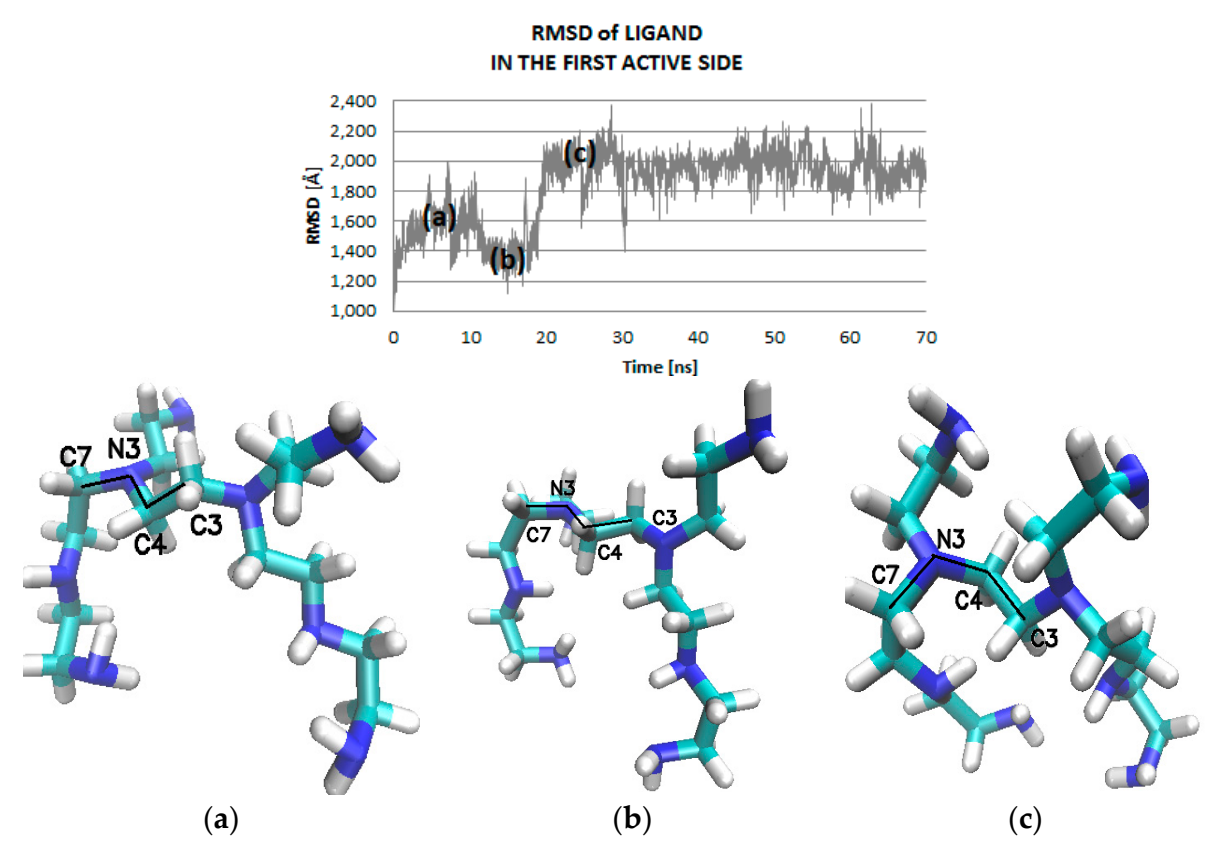

Figure 8. Distribution of values of the dihedral angle of ligand (C7-N3-C4-C3): values characterizing the ligand interaction at the first active site (LIG1) of 3QVR enzyme, inside the protein (Figure 2) during different periods of MD simulation: (a) first $10 \mathrm{~ns}$; (b) 10-20 ns; (c) 20-70 ns.

In the case of complexes located inside the enzyme body (LIG1, Figure 2), the MD simulation (Molecular Dynamic) found five amino acids: ALA287, LEU27, SER101, SER94 and SER289, involved in the creation of six HB (Figures 9 and 10) that stabilize the complex of enzyme 3QVR (Glucose oxidase) with PEI (polyethylenimine). In the case of complex located outside of the enzyme surface (LIG2, Figure 2), the first involved are ASN471, ASP358 and GLU354 (Figure 11) in the forming of the six HB (Figure 12).

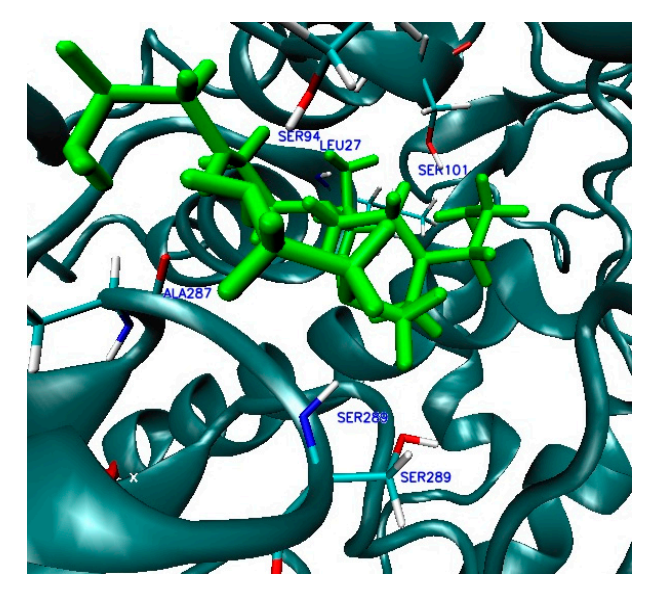

Figure 9. Interactions 3QVR enzyme-ligand PEI_C14N8_07_B22, at the first active site (LIG1). 


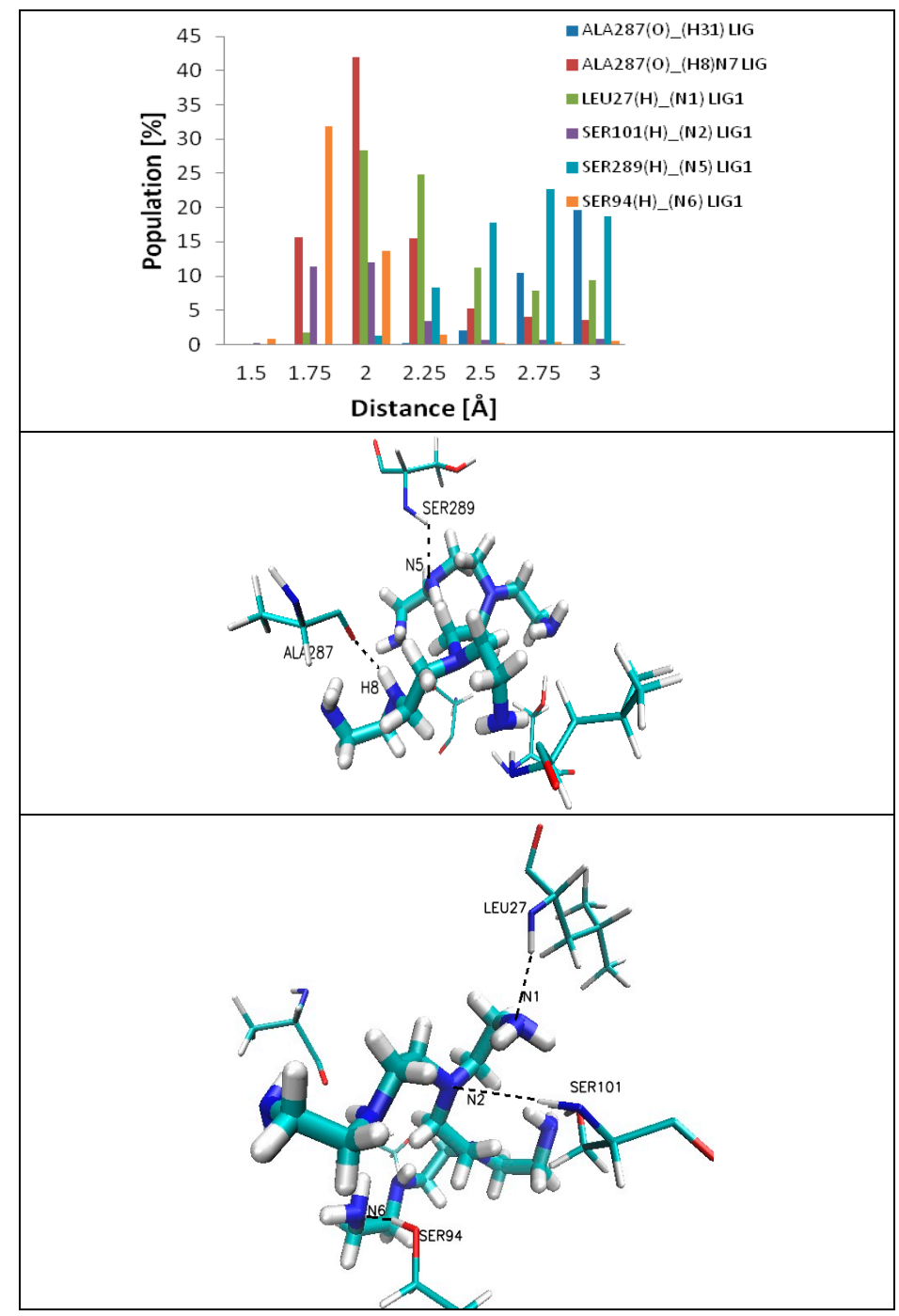

Figure 10. Distribution of the hydrogen bond lengths/interactions between PEI_C14N8_07_B22 and the amino acids at the LIG1 site of 3QVR (inside of protein), throughout the simulation time of MD.

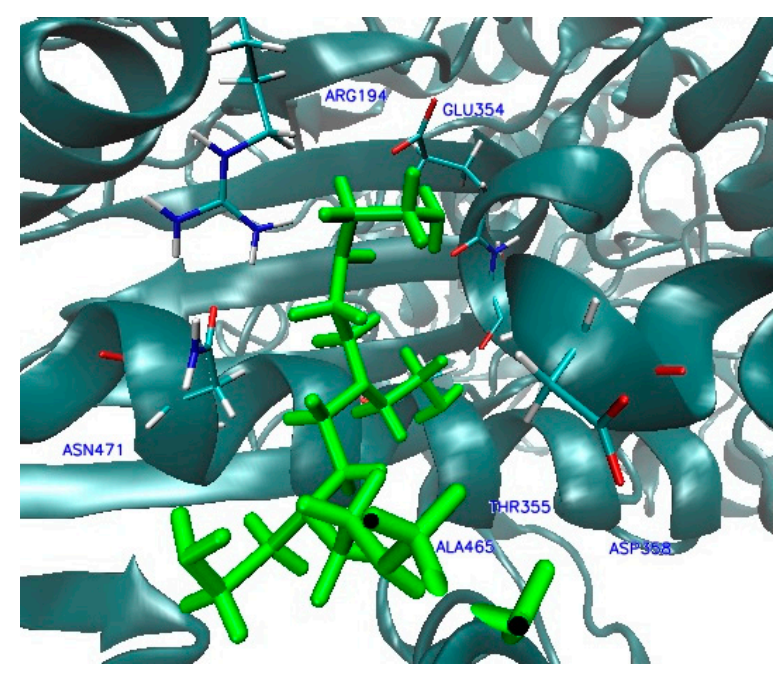

Figure 11. Interactions 3QVR enzyme-ligand PEI_C14N8_07_B22, at the second active site (LIG2). 


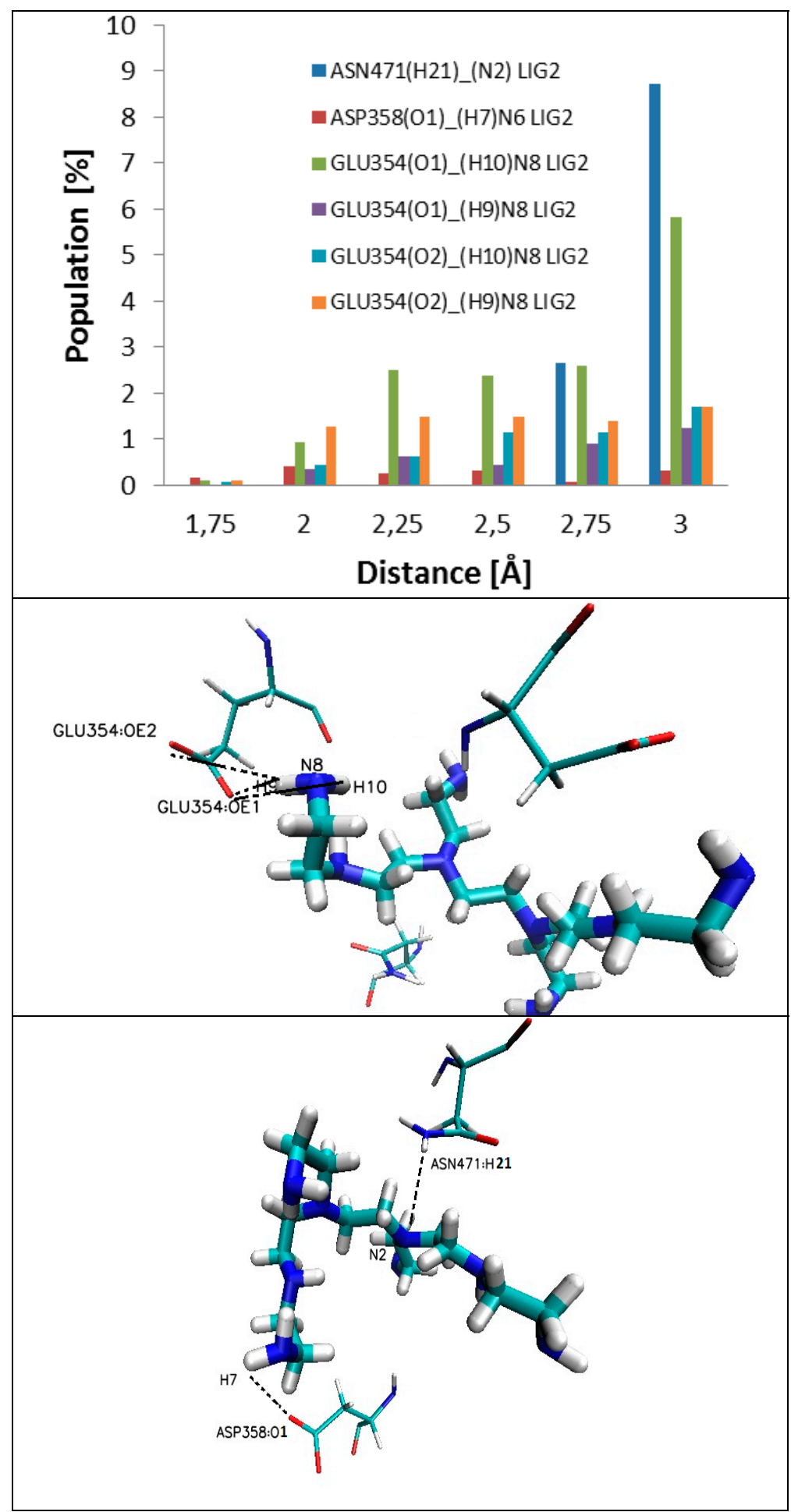

Figure 12. Distribution of the hydrogen bond lengths/interactions between PEI_C14N8_07_B22 and the amino acids at the LIG2 site of 3QVR (on the surface of protein), throughout the simulation time of MD.

The hydrogen bonds $\mathrm{Y}$... H . . X X, established between a donor $X$ and an acceptor $Y$, are classified function of the distance $d(\mathrm{Y}, \mathrm{H})$ between the acceptor $\mathrm{Y}$ and Hydrogen atom. There are "strong" $\mathrm{HB}$, with $\mathrm{d}(\mathrm{Y}, \mathrm{H})<1.6 \AA$; " average/medium", with $1.6<\mathrm{d}(\mathrm{Y}, \mathrm{H})<1.9 \AA$ and "poor/low", with $\mathrm{d}(\mathrm{Y}, \mathrm{H})>1.9 \AA$. 
With such a criterion, the protein $3 \mathrm{QVR}$ appeared to have a medium to low capacity of forming HB with the ligand PEI_C14N8_07_B22 [17] (Figures 9-13).

In the first active site LIG1 (Figure 2) of the enzymes, there are five different amino acids: ALA287, LEU27, SER101, SER289 and SER94 (Figures 9 and 10) that form HB with the ligand (PEI_C14N8_07_B22) as follows:

- ALA287(O) .... (H31-PEI)LIG1, in 30\% of all conformations, electrostatically interacts with the hydrogen atom $\mathrm{H} 31$ (of the ligand chain) to form a hydrogen bond of low strength;

- $\quad$ ALA287(O) .... .(H8-N7-PEI)LIG1, in 73\% of all conformation, makes a strong hydrogen bond (Figure 11, Table 4).

For the interaction of PEI (polyethylenimine) with other aminoacids, located either at the first side (inside the enzyme) or at the second site (outside the enzyme surface), the reader can easily use Table 4 and Figures 10 and 12.

In the case of the complex forming at the enzyme surface, the interactions ligand-enzyme appear relatively late, during MD simulations, (after about 30 and $40 \mathrm{~ns}$ ) and all of these impacts have medium and low strength. In addition, the formed bonds tend to disappear and re-appear during MD simulation (Figures 11 and 12, Table 4).

Table 4. The length distributions of the most common hydrogen bonds (electrostatic interactions) occurring between PEI_C14N8_07_B22 and selected amino acids from the LIG1 site (inside of the enzyme; left side of the table) and from the LIG2 site (on the surface of enzyme; right side of the table) of 3QVR during MD simulations.

\begin{tabular}{|c|c|c|c|c|c|}
\hline $\begin{array}{l}\text { Hydrogen } \\
\text { Bonds at LIG1 }\end{array}$ & $\begin{array}{l}\text { Hydrogen Bond } \\
\text { Length (̊̊) }\end{array}$ & $\begin{array}{l}\text { Population (Among All } \\
\text { Conformations) }(\%)\end{array}$ & $\begin{array}{c}\text { Hydrogen } \\
\text { Bonds at LIG2 }\end{array}$ & $\begin{array}{l}\text { Hydrogen } \\
\text { Bond } \\
\text { Length (̊̊) }\end{array}$ & $\begin{array}{c}\text { Population (Among } \\
\text { All Conformations) } \\
(\%)\end{array}$ \\
\hline \multirow{4}{*}{$\begin{array}{l}\text { ALA287(O)_- } \\
\text { (H31)LIG1 }\end{array}$} & 2.25 & 0.03 & $\begin{array}{c}\text { ASN471(H21) } \\
\text { (N2)LIG2 }\end{array}$ & 2.75 & 2.7 \\
\hline & 2.5 & 2.11 & ASP358(O1) & 1.75 & 0.17 \\
\hline & 2.75 & 10.48 & (H7)N6LIG2 & 2 & 0.42 \\
\hline & 3 & 19.62 & & 2 & 0.4 \\
\hline \multirow{6}{*}{$\begin{array}{l}\text { ALA287(O)_ } \\
(\mathrm{H} 8) \text { N7LIG1 }\end{array}$} & 1.75 & 15.7 & & 2.25 & 0.6 \\
\hline & 2 & 41.9 & GLU354(O1)_- & 2.5 & 0.4 \\
\hline & 2.25 & 15.5 & (H9)N8LIG2 & 2.75 & 0.9 \\
\hline & 2.5 & 5.2 & & 3 & 1.3 \\
\hline & 2.75 & 4.0 & & 1.75 & 0.1 \\
\hline & 3 & 3.6 & & 2 & 0.9 \\
\hline \multirow{6}{*}{$\begin{array}{l}\mathrm{LEU}_{27(\mathrm{H})_{-}} \\
\text {(N1)LIG1 }\end{array}$} & 1.7 & 1.8 & GLU354(O1)_ & 2.25 & 2.5 \\
\hline & 2 & 28.3 & (H10)N8LIG2 & 2.5 & 2.4 \\
\hline & 2.25 & 24.8 & & 2.75 & 2.6 \\
\hline & 2.5 & 11.2 & & 3 & 5.8 \\
\hline & 2.75 & 7.8 & & 1.75 & 0.1 \\
\hline & 3 & 9.5 & & 2 & 1.3 \\
\hline \multirow{7}{*}{$\begin{array}{l}\text { SER101(H)_- } \\
\text { (N4)LIG1 }\end{array}$} & 1.5 & 0.14 & GLU354(O2)_ & 2.25 & 1.5 \\
\hline & 1.7 & 11.31 & (H9)N8LIG2 & 2.5 & 1.5 \\
\hline & 2 & 11.94 & & 2.75 & 1.4 \\
\hline & 2.25 & 3.48 & & 3 & 1.7 \\
\hline & 2.5 & 0.63 & & 1.75 & 0.09 \\
\hline & 2.75 & 0.60 & & 2 & 0.45 \\
\hline & 3 & 0.86 & GLU354(O2)_ & 2.25 & 0.63 \\
\hline \multirow{6}{*}{$\begin{array}{l}\text { SER94(H) } \\
\text { (N6)LIG1 }\end{array}$} & 1.5 & 0.9 & (H10)N8LIG2 & 2.5 & 1.16 \\
\hline & 1.7 & 31.9 & & 2.75 & 1.16 \\
\hline & 2 & 13.7 & & 3 & 1.7 \\
\hline & 2.25 & 1.4 & & & \\
\hline & 2.5 & 0.3 & & & \\
\hline & 2.75 & 0.4 & & & \\
\hline \multirow{6}{*}{$\begin{array}{l}\text { SER289(H)_ } \\
\text { (N5)LIG1 }\end{array}$} & 3 & 0.5 & & & \\
\hline & 2 & 1.2 & & & \\
\hline & 2.25 & 8.3 & & & \\
\hline & 2.5 & 17.7 & & & \\
\hline & 2.75 & 22.7 & & & \\
\hline & 3 & 18.8 & & & \\
\hline
\end{tabular}




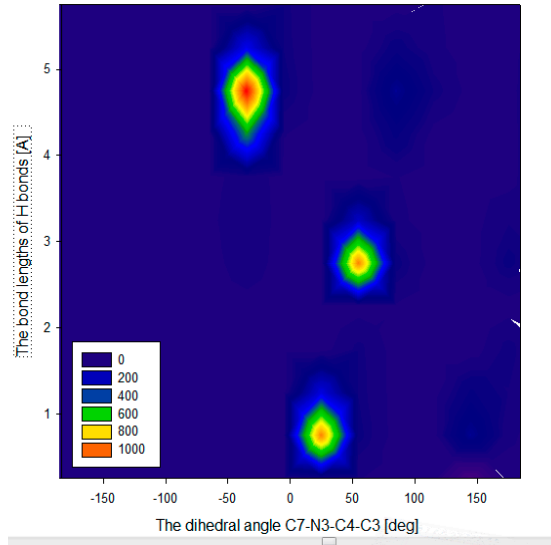

(a) ALA287(O)_LIG(H8)LIG1

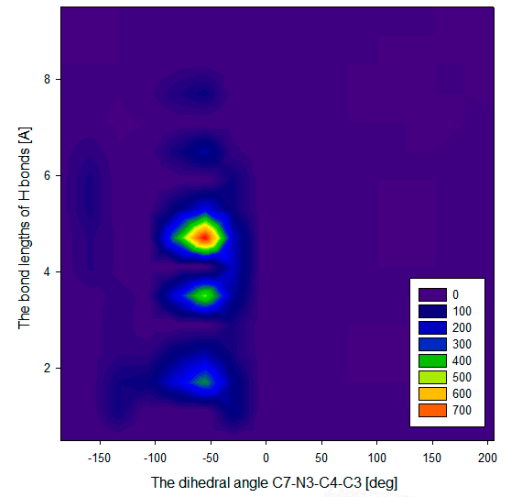

(c) SER101(H)_(N2)LIG1

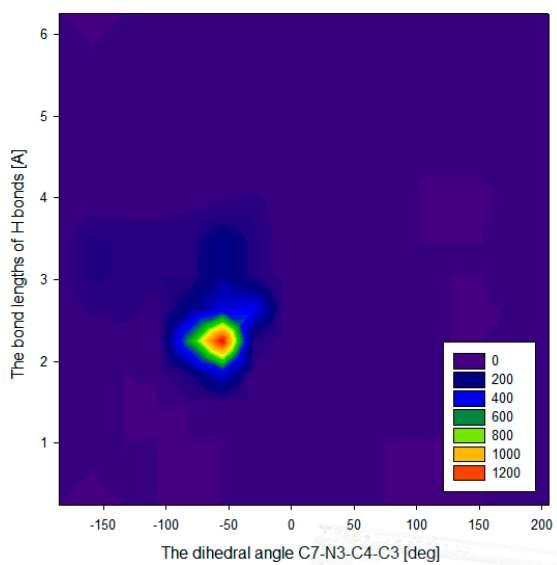

(b) LEU27(H)_(N1)LIG1

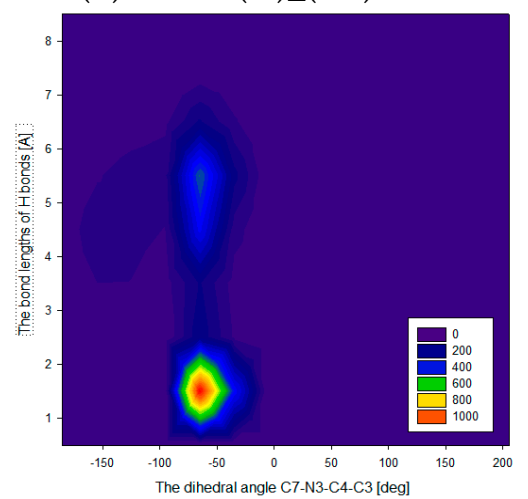

(d) SER94(H)_(N6)LIG1

Figure 13. The length of hydrogen bonds at LIG1 (inside of protein): (a) ALA287(O) ... .(H8)LIG1; (b) LEU27(H) . . . .(N1)LIG1; (c) SER101(H) . . . (N2)LIG1; (d) SER94(H) . . . (N6)LIG1 as a function of dihedral angle C7-N3-C4-C3 during all the time of MD simulation.

Note that the appearance/disappearance of hydrogen bonds is closely related to the values of dihedral angle C7-N3-C4-C3 of the ligand PEI_C14N8_07_B22 (Figures 8 and 13) in the complex at LIG1. In the case of ALA287(O) ... .LIG(H8)LIG1, while the angle C7-N3-C4-C4 reaches the value +50 degree $[\mathrm{deg}]$, the strongest hydrogen bonds (with lengths around $1.65 \AA$-Figure 13a) are formed. However, the increase of the dihedral angle values from 50 up to 100 degree [deg] is associated with an increased length of the hydrogen bonds, from $2.5 \AA$ to $3 \AA$, meaning a decrease of $\mathrm{HB}$ strength with the appearance of hydrogen bonds of medium and low strength. For the interaction LEU27(H) ... . (N1)LIG1, the value of the dihedral angle C7-N3-C4-C4 is about -50 degree [deg] and the hydrogen bond reaches a length of about $2.2 \AA$ (Figure 13b). Here, corresponding to the interval -25 to -100 degree $[\mathrm{deg}]$ of dihedral angle, there appear hydrogen bonds of length ranging from $1.8 \AA$ to $2.5 \AA$. The bond SER101(H) ... . . . (N2-PEI)LIG1 periodically reaches a length of $1.8 \AA$ (Figure 13c). In the case of SER94(H) ... .(N6)LIG1, such interactions are characterized as strong and medium while the values of dihedral angle ranged from -50 to -90 degree [deg] (Figure 13d).

As one can see, the stabilization of ligand-protein complexes is related to the strength and number of hydrogen bonds created between them. This interaction can be characterized by the values of calculated enthalpy contribution of Gibbs Free Energy; in the two cases of complex PEI-enzymes, located inside of the protein (LIG1) and on its surface (LIG2), the values of enthalpy are $-12.486 \pm 8.7321$ and $-13.7496 \pm 6.7161$, respectively. 


\section{Materials and Methods}

During the docking simulation, the whole area of the enzyme was mapped, by changing the parameters of grid box step by step; the crystal structures of 3QVR were used, as downloaded from the Brookhaven Protein Database (PDB) [18,19]. A Molecular Dynamics (MD) procedure was applied to the two best-docked enzyme-ligand (3QVR-PEI_C14N8_07_B22) complexes. The ligand structure was characterized by using the Amber force field parameters; the atomic charges were calculated according to the Merz-Kollmann scheme, via the RESP procedure [20] at HF/6-31G* level of theory. Each system was neutralized and immersed in a periodic TIP3P (Transferable Intermolecular Potential 3P) water box (Jorgensen et al. 1983). The considered systems were heated up to $300 \mathrm{~K}$ by 100 ps of initial MD simulation, while the temperature was controlled by the Langevin thermostat (IN\&NY, USA) [21]. The periodic boundary conditions and SHAKE algorithm [22] were applied to $70 \mathrm{~ns}$ of MD simulation, the first $10 \mathrm{~ns}$ of the simulation time being used for the equilibration of the system; the next $60 \mathrm{~ns}$ of trajectory were used in the effective analysis of interaction between the considered subunits. Structural analysis was performed by the VMD (Visual Molecular Dynamic) package [23]. The energetic characterization of the interaction ligand-enzyme active site was obtained by the Molecular Mechanic/Poisson-Boltzmann Surface Area (MMPBSA) method [24]. In all MD simulations, the AMBER 11 package (San Francisco, CA, USA) [25] was used.

\section{Conclusions}

Glucose oxidase (GOx) is an enzyme that has found several commercial applications in chemical and pharmaceutical industries including biosensors that use this enzyme immobilized onto different nanomaterials and polymers such as polyethylenimine (PEI). The present data for the first time describe a more specific and oriented interaction of glucose oxidase and PEIs which can serve in different bioconjugation processes, and, at the same time, this part of the work is a proposition to continue the incipient study with other shapes/sizes of PEI. The problem of GOx immobilization on PEI is to retain the enzyme native activity despite its immobilization onto the polymer surface. Therefore, an $\mathrm{MD}$ study of the PEI ligand (C14N8_07_B22) and GOx enzyme (3QVR) was performed to investigate the stability of the complex C14N8_07_B22-3QVR and the affinity of the PEI ligand to the docking sites of GOx. Analysis of the dynamic behavior of complexes formed by the ligand PEI_C14N8_07_B22 with 3QVR inside of the enzyme body and on its surface, respectively, revealed important differences in their structural and energetic characteristics. For the both complexes, conformations stabilized by hydrogen bonds were observed during the docking stage. The docking procedure showed two sites of major interaction of the protein with the polymer PEI: (LIG1), of $-5.8 \mathrm{kcal} / \mathrm{mol}$ and (LIG2), of $-4.5 \mathrm{kcal} / \mathrm{mol}$, located inside the enzyme and on its surface, respectively. Docking also found domains of protein that exhibit no interactions with the ligand or have even repulsive characteristics. The results obtained during docking became the starting point in molecular dynamics simulation. The RMSD (Root-Mean-Square Deviation) values of systems, their average values and standard deviations show that these two systems (LIG1 and LIG2) are quite similar. In the first active side, inside of the protein, after $20 \mathrm{~ns}$ of MD (Molecular Dynamic), stabilization values of RMSD of ligand appears, with poor fluctuations from 1.6 to $2.0 \AA$. The average values of RMSD of ligand show significantly higher mobility of PEI (polyethylenimine) on the surface of protein (at LIG2) compared to that inside of enzyme (at LIG1). The mobility of the ligand inside of the protein is correlated with changes in the values of dihedral angle (C7-N3-C4-C3). The hydrogen bonds (i.e., interactions) formed between enzyme and ligand, are shorter (i.e., stronger) inside of the protein compared to $\mathrm{HB}$ on the protein surface. The lengths of the hydrogen bonds ligand-enzyme created on the surface of protein are long and hence $\mathrm{HB}$ and the corresponding interactions are weak.

Acknowledgments: This work was financially supported by the GEMNS project granted in the European Union's Seventh Framework Programme under the frame of the ERA-NET EuroNanoMed II (European Innovative Research and Technological Development Projects in Nanomedicine) and BSZ acknowledges the Grant No. 237 PCSS (Poznań, Poland). 
Author Contributions: The research was conceived by Beata Szefler, Mircea V. Diudea, Mihai V. Putz, and Ireneusz P. Grudzinski. The computational work was performed by Beata Szefler. Beata Szefler extracted, processed and analysed the data, and drafted the manuscript. Beata Szefler, Mircea V. Diudea, Mihai V. Putz and Ireneusz P. Grudzinski supervised and coordinated the project and revised the manuscript. All authors participated in project discussion. All authors read and approved the final manuscript.

Conflicts of Interest: The authors declare no conflict of interest.

\section{References}

1. Sittiwet, C.; Srisa-ard, M.; Baimark, Y. Immobilization of Glucose oxidase and Peroxidase onto Nanoporous Chitosan-based Films. Malays. Polym. J. 2010, 5, 108-116.

2. Homma, T.; Ichimura, T.; Kondo, M.; Kuwahara, T.; Shimomura, M. Covalent immobilization of glucose oxidase on the film prepared by electrochemical polymerization of $N$-phenylglycine for amperometric glucose sensing. Eur. Polym. J. 2014, 51, 130-135. [CrossRef]

3. Shimomura, M.; Kojima, N.; Oshima, K.; Yamauchi, T.; Miyauchi, S. Covalent Immobilization of Glucose Oxidase on film prepared by electrochemical copolymerization of Thiophene-3-acetic acid and 3-Methylthiophene for Glucose sensing. Polym. J. 2001, 33, 629-631. [CrossRef]

4. RCSB PDB. GOx-Molecule of the Month. Available online: http://www.rcsb.org/pdb/home/home.do (accessed on 23 December 2008).

5. Pekel, N.; Salih, B.; Guven, O. Enhancement of stability of glucose oxidase by immobilization onto metal ion-chelated poly(N-vinyl imidazole) hydrogels. J. Biomater. Sci. Polym. Ed. 2005, 16, 253-266. [CrossRef] [PubMed]

6. Karachevtsev, V.A.; Glamazda, A.Y.U.; Zarudnev, E.S.; Karachevtsev, M.V.; Leontiev, V.S.; Linnik, A.S.; Lytvyn, O.S.; Plokhotnichenko, A.M.; Stepanian, S.G. Glucose oxidase immobilization onto carbon nanotube networking. Ukr. J. Phys. 2012, 57, 700-709.

7. Sharma, K.P.; Zhang, Y.; Thomas, M.R.; Brogan, A.P.S.; Perriman, A.W.; Mann, S. Self-organization of glucose oxidase-polymer surfactant nano-constructs in solvent-free soft solids and liquids. J. Phys. Chem. B 2014, 118, 11573-11580. [CrossRef] [PubMed]

8. Poplawska, M.; Bystrzejewski, M.; Grudzinski, I.P.; Cywinska, M.A.; Ostapko, J.; Cieszanowski, A. Immobilization of gamma globulins and polyclonal antibodies of class IgG onto carbon-encapsulated iron nanoparticles functionalized with various surface linkers. Carbon 2014, 74, 180-194. [CrossRef]

9. Karajanagi, S.S.; Vertegel, A.A.; Kane, R.S.; Dordick, J.S. Structure and function of enzymes adsorbed onto single-walled carbon Nanotubes. Langmuir 2004, 20, 11594-11599. [CrossRef] [PubMed]

10. Feng, W.; Ji, P. Enzymes immobilized on carbon Nanotubes. Biotechnol. Adv. 2011, 29, 889-895. [CrossRef] [PubMed]

11. Vicent, M.J.; Duncan, R. Polymer conjugates: Nanosized medicines for treating cancer. Trends Biotechnol. 2006, 24, 39-47. [CrossRef] [PubMed]

12. Kasprzak, A.; Popławska, M.; Bystrzejewski, M.; Łabędź, O.; Grudziński, I.P. Conjugation of polyethylenimine and its derivatives to carbon-encapsulated iron nanoparticles. RSC Adv. 2015, 5, 85556-85567. [CrossRef]

13. Herlem, G.; Lakard, B.; Fahys, B. Recent research developments in electroanalytical chemistry. Trivandrum 2001, 3, 21-33.

14. Herlem, G.; Lakard, B. Ab initio study of the electronic and structural properties of the crystalline polyethyleneimine polymer. J. Chem. Phys. 2004, 120, 9376-9382. [CrossRef] [PubMed]

15. Padilla-Martíneza, S.G.; Martínez-Jothar, L.; Sampedro, J.G.; Tristan, F.; Pérez, E. Enhanced thermal stability and $\mathrm{pH}$ behavior of glucose oxidaseon electrostatic interaction with polyethylenimine. Int. J. Biol. Macromol. 2015, 75, 453-459. [CrossRef] [PubMed]

16. Todde, G.; Hovmoller, S.; Laaksonen, A.; Mocci, F. Penicillium amagasakiense: Characterization of the transition state of its denaturation from molecular dynamics simulations. Proteins 2014, 82, 2353-2363. [CrossRef] [PubMed]

17. Cleland, W.W.; Kreevoy, M.M. Low-barrier hydrogen bonds and enzymic catalysis. Science 1994, 264, 1887-1890. [CrossRef] [PubMed]

18. Berman, H.M.; Henrick, K.; Nakamura, H. Announcing the worldwide Protein Data Bank. Nat. Struct. Mol. Biol. 2003, 10, 980. [CrossRef] [PubMed] 
19. PubChem database. Available online: https://pubchem.ncbi.nlm.nih.gov/search (accessed on 5 October 2014).

20. Bayly, C.I.; Cieplak, P.; Cornell, W.D.; Kollman, P.A. A well-behaved electrostatic potential based method using charge restraints for deriving atomic charges: the RESP model. J. Phys. Chem. 1993, 97, 10269-10280. [CrossRef]

21. Adelman, S.A.; Doll, J.D. Generalized Langevin equation approach for atom/solid surface scattering: General formulation for classical scattering off harmonic solids. J. Chem. Phys. 1976, 64, 2375-2388. [CrossRef]

22. Ryckaert, J.P.; Ciccotti, G.; Berendsen, H.J.C. Numerical integration of the Cartesian equations of motion of a system with constraints: molecular dynamics of n-Alkanes. J. Comput. Phys. 1977, 23, 327-341. [CrossRef]

23. Humphrey, W.; Dalke, A.; Schulten, K. VMD: Visual molecular dynamics. J. Mol. Graph. 1996, 14, 33-38. [CrossRef]

24. Miller, B.R.; McGee, T.D.; Swails, J.M.; Homeyer, N.; Gohlke, H.; Roitberg, A.E. MMPBSA.py: An efficient program for End-State free energy calculations. J. Chem. Theory Comput. 2012, 8, 3314-3321. [CrossRef] [PubMed]

25. Case, D.A.; Darden, T.A.; Cheatham, T.E., III; Simmerling, C.L.; Wang, J.; Duke, R.E.; Luo, R.; Walker, R.C.; Zhang, W.; Merz, K.M.; et al. AMBER 11. Available online: http:/ /ambermd.org (accessed on 11 February 2016).

(C) 2016 by the authors; licensee MDPI, Basel, Switzerland. This article is an open access article distributed under the terms and conditions of the Creative Commons Attribution (CC-BY) license (http://creativecommons.org/licenses/by/4.0/). 